\title{
Synthesis maps for multivariate ordinal variables in manufacturing
}

\author{
G. BRONDINO + F. FRANCESCHINI*†, M. GALETTO $\dagger$ and G. VICARIO
}

$\dagger$ DISPEA, Politecnico di Torino, Corso Duca degli Abruzzi 24, 10129, Torino, Italy

\$DIMAT, Politecnico di Torino, Corso Duca degli Abruzzi 24, 10129, Torino, Italy

(Revision received December 2005)

\begin{abstract}
Many quality characteristics of products or services are commonly evaluated on ordinal scales with a finite number of categories. A systematic analysis of categorical variables collected over time may be very useful for a profitable management strategy. In order to measure customer satisfaction or quality improvement in a process, two or more quality characteristics are often conjointly measured and summarized by suitable indexes. A common practice suggests evaluating a synthetic index by mapping each outcome of a multivariate ordinal variable into numbers. This procedure is not always legitimate from the measurement theory point of view. In this paper an alternative approach based on the algebraic theory of the ordered sets is proposed. This method avoids mapping multivariate components into numbers. Multivariate ordinal variable components are synthesized by ordering the multivariate sample space. The ordering criterion is defined on the basis of the specific characteristics of the process at hand. Practical effects in the use of this method are shown on a series of application examples.
\end{abstract}

Keywords: Multivariate ordinal variables; Statistical process control; Multidimensional ordinal structures; Synthesis maps; Ranking and ordering; Lattice theory

\section{Introduction}

In assessing the characteristics of a product, many practical problems involve the use of ordinal scales with a finite number of categories. The levels of these scales are terms such as good, bad, medium, etc., which can be ordered according to the specific meaning of the characteristic at hand.

When ordinal data are obtained by evaluating at the same time two or more features of the same product, we are in presence of a 'multidimensional ordinal structure'.

This kind of structure is widely employed both in social and behavioural sciences and in decision-making applications. In making choices, for example, it is reasonable to have to compare different alternatives with a variety of ordinal attributes or dimensions (Roberts 1979, Roy and Bouyssou 1993, Moshkovich et al. 2002).

Multidimensional ordinal structures are also considered in the field of quality control when a series of attributes of a product are measured by linguistic or

*Corresponding author. Email: fiorenzo.franceschini@polito.it 
qualitative scales (Clifford 1959, Liu and Singh 1993, Liu et al. 1999, Taleb and Limam 2002). The conjoint measure of quality characteristics (i.e. comfort, design, sturdiness, etc.) of a manufacturing product is a typical multidimensional problem. The same problem occurs when measuring the quality of a service (Parasuraman et al. 1985).

From an algebraic point of view, a multidimensional ordinal structure may be seen as the product structure of a number of simpler one-dimensional ordinal sets. In literature different approaches have been developed for managing complex multidimensional structures. The area of the measurement theory interested in the simultaneous measurement of composite products is commonly called conjoint measurement (Krantz et al. 1971, Roberts 1979). The problem of aggregating and ordering multivariate ordinal data has been widely studied also in statistics (Tukey 1975, Agresti 2002).

A common practice consists of evaluating synthesis indices by mapping multivariate ordinal data into numbers. Nevertheless, this procedure is not always legitimate from the measurement theory point of view (Roberts 1979). Moreover, the sample dimension and the exiguous number of scale levels can cause some restriction in their application.

The aim of this paper is to propose a general approach for data aggregation and management of multidimensional ordinal problems, based on the algebraic theory of the ordered set (lattice theory) (Donnellan 1968). Product structures may be transformed into simpler ordered sets by suitable map-functions established by the decision-makers according to the specific characteristics of the process at hand.

Based on this approach, two kinds of problems can be faced:

1. The aggregation of a sample of results for a univariate ordinal variable (Franceschini et al. 2005).

2. The synthesis of different components of a multivariate ordinal variable (Franceschini and Galetto 2001).

The fundamentals of the lattice theory and its applications to the multidimensional ordinal structures are discussed in section 2 . The problems of the aggregation of a sample of results for a univariate ordinal variable, and the synthesis of multivariate ordinal data, respectively, are analysed in sections 3 and 4. Results obtained applying lattice theories are compared with the 'classical' methods (mapping of ordinal variables into numbers). The paper provides many illustrative examples of practical effects of both approaches.

\section{Ranking and lattice theory}

The multidimensional ordinal structures can be represented by the algebraic theory of the ordered sets (Donnellan 1968). After a brief description of the ordering theory applied to multidimensional ordinal structures, this methodology will be used for the definition of a ranking of elements belonging to a finite multidimensional ordinal sample space.

Definition: Let us consider a finite ordered set $P ; P$ is a chain (or a totally ordered set) if, for all $x, y \in P$, either $x \leq y$ or $y \leq x$, with $x, y$ any two categories of 
an ordinal variable $X$ (i.e. any two elements of $P$ are comparable) (Davey and Priestley 1990).

Usually, a finite ordered set is commonly represented by a Hasse diagram: a configuration of nodes (the elements of the set) and arcs, indicating the covering relation (Roberts 1979). So it is easy to detect from the Hasse diagram (see figure 1) whether a category of $X$ is lower than another: $x$ (low) is lower than $y$ (high) if there is a sequence of connected line segments moving upwards from $x$ to $y$. Two categories are not comparable if no arc is drawn between them in a Hasse diagram.

Now, let consider two ordinal variables $X$ and $Y$ : the former measures the noise of a car engine according to three categories (high, medium and low), the latter measures the noise of a car fan with four categories (moderately noisy, quite noisy, significantly noisy and very noisy). Let $(X, Y)$ be the bivariate ordinal variable that measures conjointly the noise of the car engine and of the car fan. The possible outcomes of $(X, Y)$ are the elements of the Cartesian product of the two category sets $X$ and $Y$. The order set theory states that the Cartesian product of two chains is still an ordered structure, but it is not a chain. Some elements are not comparable with each other (see figure 2).

Definition: A partially ordered set (poset) is a set $P$ characterized by a binary order relation. It is not required that any two elements of $P$ are comparable.

If $x$ and $y$ are two elements of a poset $P$, even if they cannot be comparable with each other, for both of them we can identify an element of $P$ which is equivalent or greater with respect to the ordered relation of $P$. A finite poset turns into a chain when all its elements are pairwise comparable.

Definition: A complete lattice $P$ is a poset in which the supremum (the least upper bound) and the infimum (the greatest lower bound) exist for all subsets of $P$.

The finite set of possible outcomes of the bivariate ordinal variable $(X, Y)$ in figure 2 is a complete lattice. In other words, the Cartesian product of $X$ and $Y$ is intrinsically ordered (it sounds to affirm that a 'medium noisy' of the engine and a 'quite noisy' of the fan is minus a 'high noisy' of the engine and a 'significant noisy'

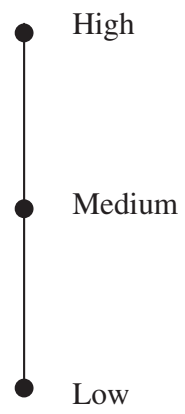

Figure 1. Hasse diagram of an ordinal variable $X$, defined on a three categories scale: low, medium and high. 


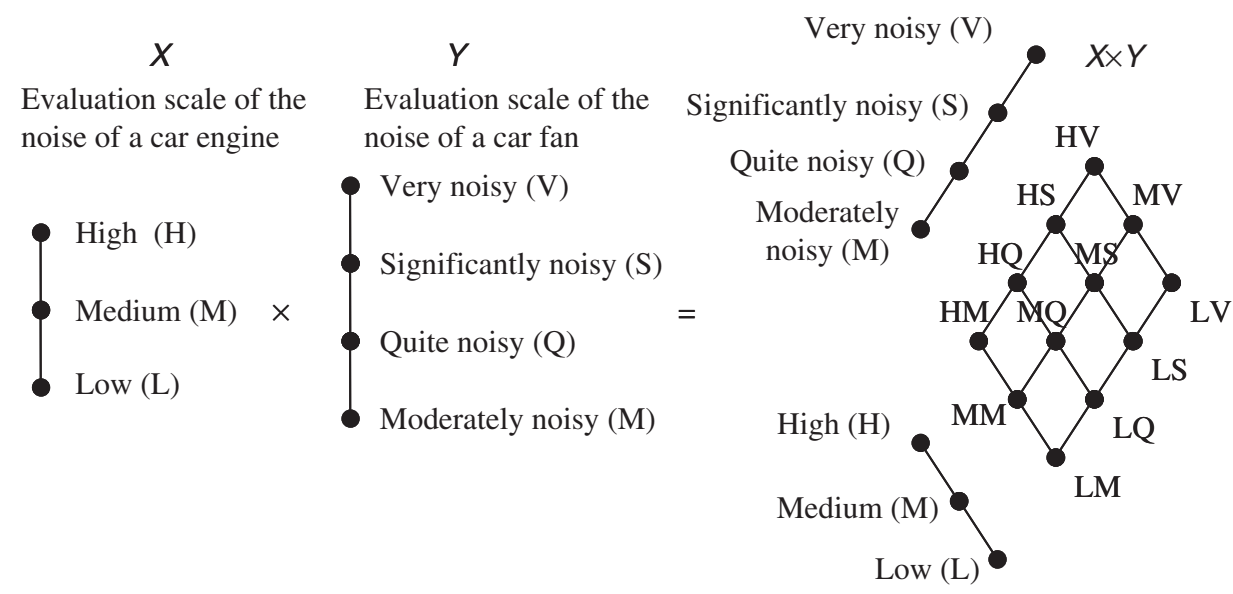

Figure 2. Hasse diagrams for the categories of the ordinal variables $X$ (noise, car engine) and $Y$ (noise, car fan). The set of possible outcomes of the bivariate ordinal variable $(X, Y)$ is the Cartesian product $X \times Y$.

of the fan). Unfortunately, the intrinsic order does not imply the total ordering of the set. Some elements are non-comparable: for example, the outcomes 'medium, quite noisy' and 'low, very noisy'.

In some cases, however, the elements of a non-comparable pair of outcomes are not equally important, from a decision-making point of view. For example, if the variable $X$ is more influent than $Y$, the outcome 'low', 'very noisy' is probably preferable to 'medium', 'quite noisy'. In such cases the process 'decision-maker' can adopt a map function which assigns to any outcomes of the multivariate ordinal variable a specific ranking (Davey and Priestley 1990).

Definition: Let $P$ indicate the complete lattice of all possible outcomes of a multivariate ordinal variable $\left(X_{1}, X_{2}, \ldots, X_{n}\right)$ and let $Q$ be a finite poset, a map function is defined as $\varphi: P \rightarrow Q$ (see figure 3 ).

A ranking of all possible outcomes of a multivariate ordinal variable $\left(X_{1}, X_{2}, \ldots, X_{n}\right)$, ordered according to the structure of $P$, can be defined by means of the $Q$ structure. The co-domain $(Q)$ of the map is a complete lattice (or even a chain).

Definition: Let $\left(x_{1}, x_{2}, \ldots, x_{n}\right)$ and $\left(y_{1}, y_{2}, \ldots, y_{n}\right)$ be any couple of outcomes of $\left(X_{1}, X_{2}, \ldots, X_{n}\right)$, then a map is said to be order-preserving if $\left(x_{1}, x_{2}, \ldots, x_{n}\right) \leq_{P}$ $\left(y_{1}, y_{2}, \ldots, y_{n}\right)$ implies $\varphi\left(x_{1}, x_{2}, \ldots, x_{n}\right) \leq_{Q} \varphi\left(y_{1}, y_{2}, \ldots, y_{n}\right)$, where $\leq_{P}$ and $\leq_{Q}$ are respectively the order relations on $P$ and $Q$.

To represent some processes, it may be reasonable to adopt a monotone map function to link $P$ with $Q$; see figure $3(\mathrm{~b})$.

Definition: A monotone map function is an order-preserving map defined on a complete lattice of all possible outcomes of an ordinal multivariate variable $\left(X_{1}, X_{2}, \ldots, X_{n}\right)$. 


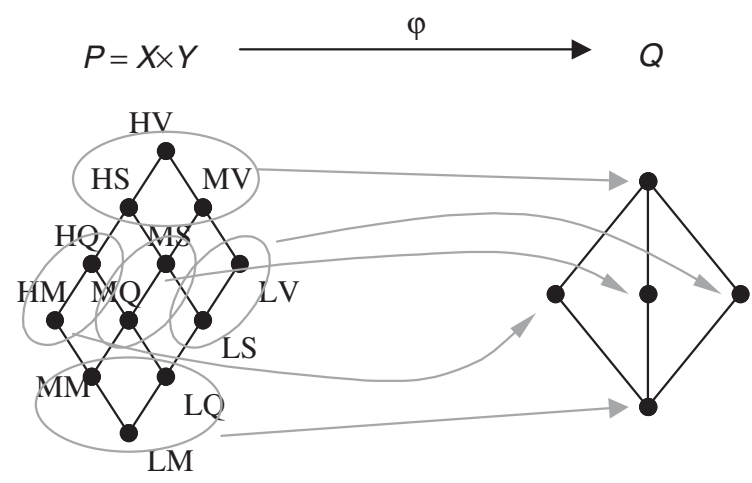

(a)

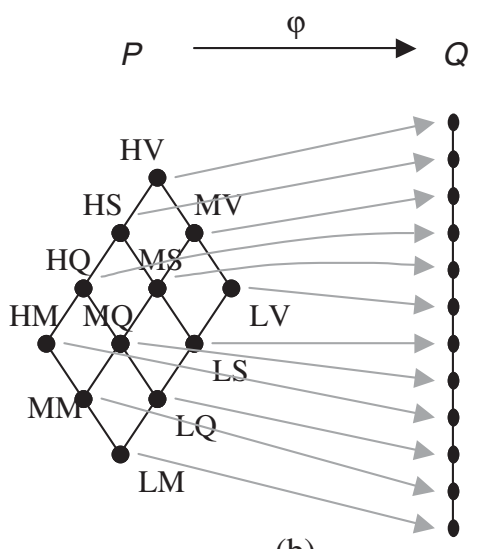

(b)

Figure 3. An example of a map function (a), and of a monotone map function (b). The figure shows the finite set of all possible outcomes of the bivariate ordinal variable $(X, Y)$ noise evaluation of a car engine and a car fan, to the poset $Q$ (L, low; M, medium; H, high; M, Moderately noisy; Q, quite noisy; S, significantly noisy; V, very noisy).

It is worthwhile stressing that a map function may also be no-order preserving. Let us consider, for example, a company which is performing a test on the air conditioning plant of its offices. The aim is to evaluate the performance of the plant as it is perceived by the personnel. Each employee is asked to express his/her judgement about the perceived humidity $(X)$ and temperature $(Y)$. Evaluations are given on a three-level scale: 'low', 'medium' and 'high'.

If we refer to the domain space $P$ of the two-dimensional variable $(X, Y)$, HH dominates MM.

On the other hand, the most favourable condition is the one scoring 'medium' both for the humidity and for the temperature (i.e. MM). This situation is certainly preferable to the case in which both the temperature and the humidity are 'high' (i.e. $\mathrm{HH}$ ).

The resulting ordering in the co-domain space, after the application of the plant manager map-function, implies that $\mathrm{MM}$ has to be preferred to $\mathrm{HH}$. Therefore, from the plant manager's point of view, the adopted map function is non monotone (see figure 4).

\section{Synthesis maps for univariate ordinal variable}

Many problems referring to the aggregation of a sample of outcomes from a univariate ordinal variable can be rigorously analysed by lattice theory.

The approach is organized in five steps:

1. Sample definition.

2. Construction of an appropriate map function for aggregating the elements of each single sample; such a function must be defined on the basis of the specific 


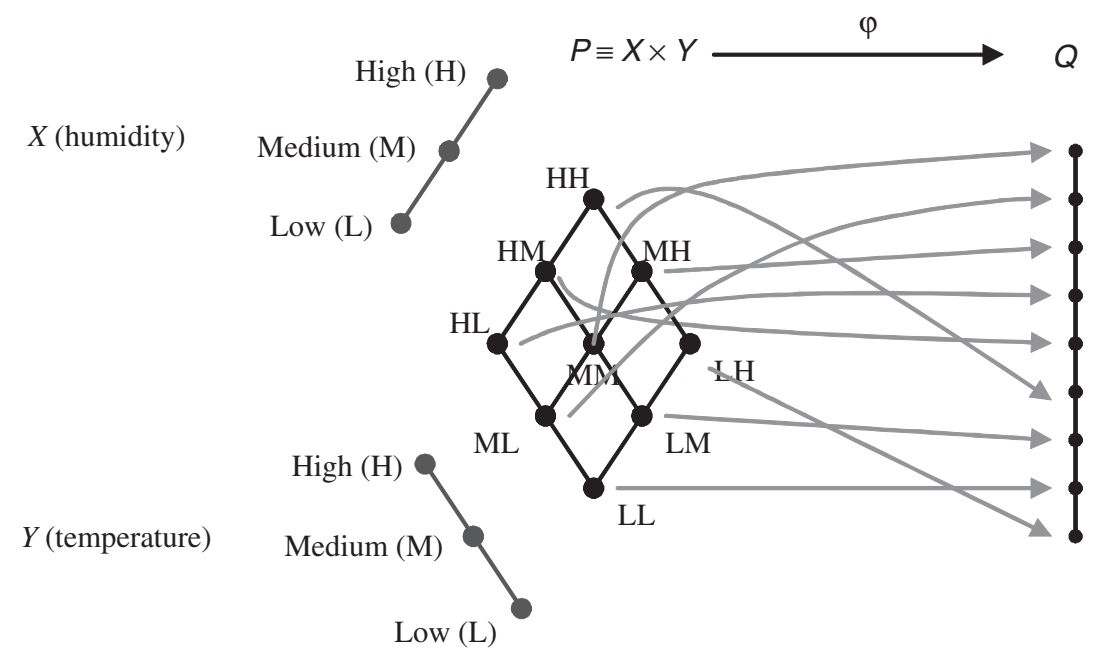

Figure 4. Hasse diagrams of the categories of the ordinal variables $X$ and $Y$. The non-monotone map function $\varphi$, defined by the plant manager according to the specific characteristics of the process at hand, maps the set of all possible outcomes $X \times Y$ into the chain $Q$.

characteristics of the process and without introducing improper (numerical) codifications.

3. Representation by a Hasse diagram.

4. Check of mathematical properties of the new ordinal variable defined in the map function co-domain.

5. Analysis of results.

Let us consider, for example, the comparison of the performances of two different manufacturing lines for the production of a mechanical component. The comparison is carried out by evaluating the quality of the surface finishing of each component by a visual control. For both treatments, every hour a sample of five parts is analysed. The evaluation is given on a three-level scale: 'low', 'medium' and 'high'.

The problem is synthesizing the information contained in the samples in order to state which of the two processes is the most worthwhile. In this case the considered ordinal variable is $X$, defined as the 'product surface finishing'. The levels of variable $X$ are the total ordered set (chain) $X$ : $\{$ low (L), medium (M), high $(\mathrm{H})\}$. Each sample of five elements can be seen as an element of the Cartesian product

$$
\prod_{i=1}^{5} X_{i} \text { (sample space). }
$$

This sample space can be represented by a $3^{5}$ complete lattice.

In order to define an appropriate index for ordering the sample space elements, a subset $P^{\prime}$ of this space including all possible combinations of five elements of $X$ is considered (see figure 5).

In $P^{\prime}$, all permutations of the same element combination are represented by a unique reference sample. For example, HMHML and LHHMM are represented by the sample HHMML. Subset $P^{\prime}$ is a complete lattice (see figure 5). 


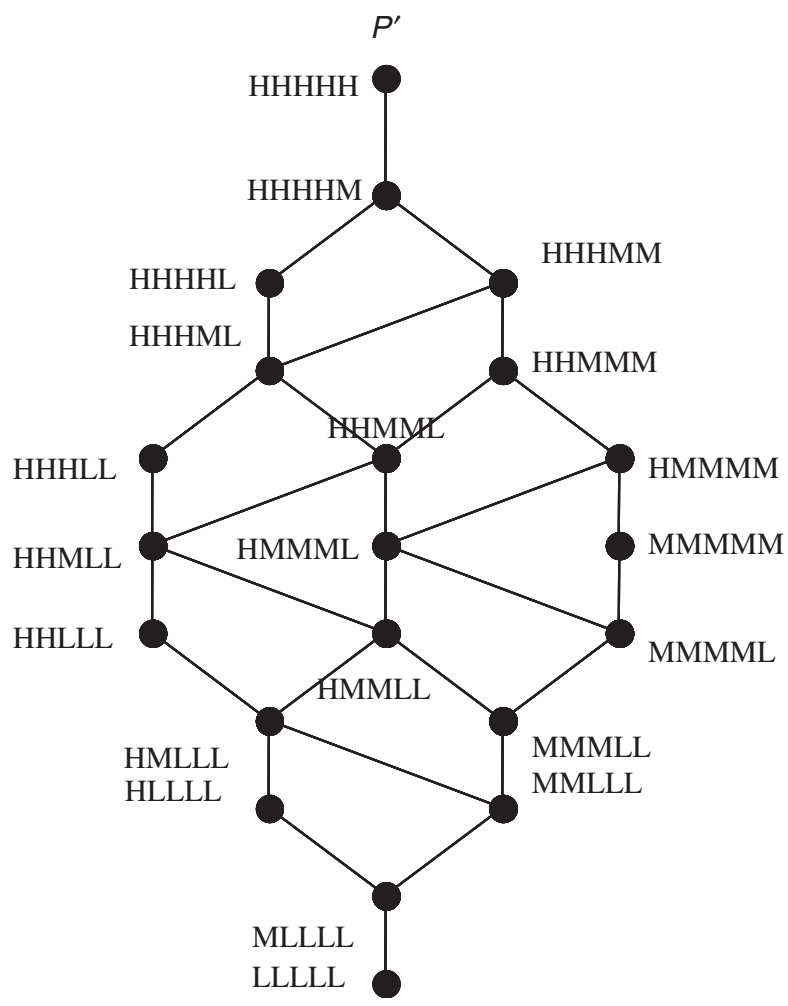

Figure 5. Reduced poset $P^{\prime}$ (complete lattice) obtained by the contraction of the sample space $\left(3^{5}\right.$ elements) of the variable $X$ for the evaluation of a 'product surface finishing'.

Table 1. Ten samples of size 5 of the qualitative variable $X$ drawn from two manufacturing lines, namely process $\alpha$ and process $\beta$. Evaluations are given on a three-level scale: $\mathrm{H}$, high; M, medium; L, low.

\begin{tabular}{lll}
\hline Sample number & Process $\alpha$ & Process $\beta$ \\
\hline 1 & HHMMM & HHLLL \\
2 & HHHHL & HHMMM \\
3 & HHMMM & HMMMM \\
4 & HHHML & HHMMM \\
5 & HHMMM & HHHHL \\
6 & HHHML & HHHHH \\
7 & HHMMM & HHHMM \\
8 & HHHMM & HHMMM \\
9 & HHHHL & HHMMM \\
10 & HHHHM & HHHHM \\
\hline
\end{tabular}

Table 1 shows the results of ten subsequent samples extracted from two manufacturing lines, named process $\alpha$ and process $\beta$.

As a first attempt to compare these two series of samples, a numerical codification is introduced. This codification is arbitrary. Different codifications determine different sample rank order (Franceschini et al. 2005). 


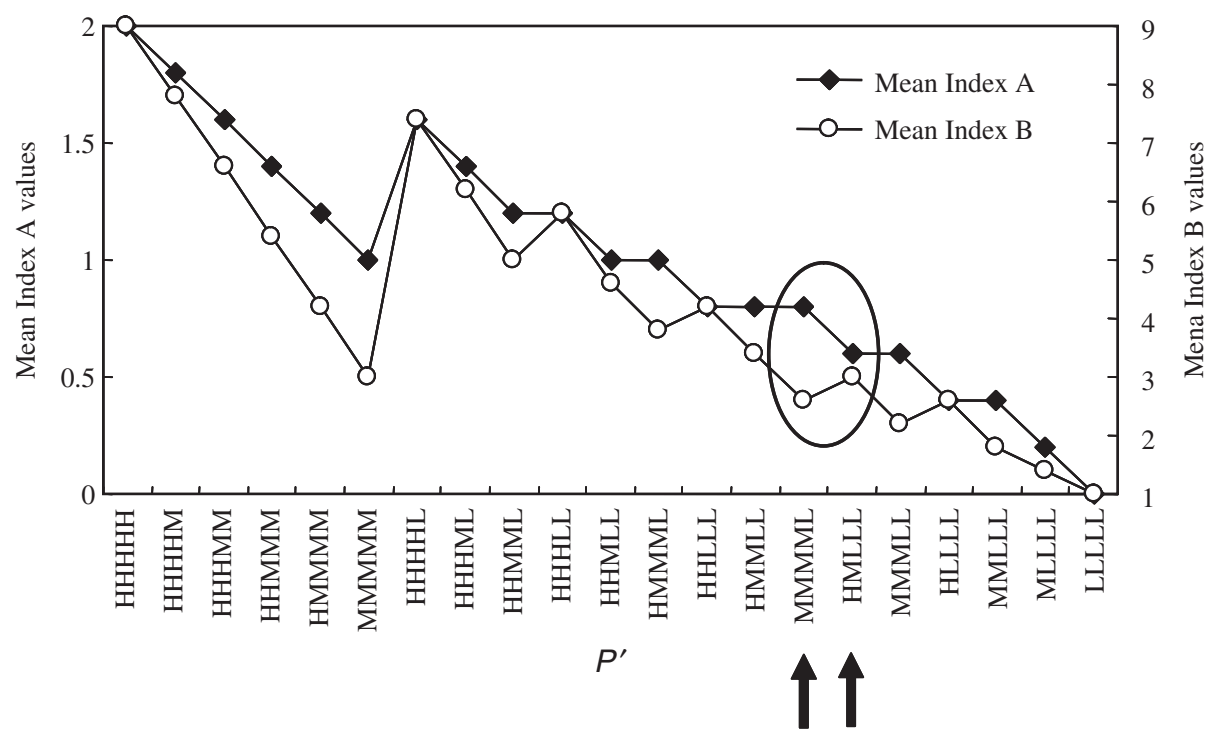

Figure 6. Mean indices A (low, 0; medium, 1; high, 2) and B (low, 1; medium, 3; high, 9) evaluated on the combination space $P^{\prime}$ for a sample size $=5$. The levels of the ordinal variable $X$ are $\mathrm{L}$, low; $\mathrm{M}$, medium; $\mathrm{H}$, high.

Let us consider, for example, two codifications:

$$
\begin{aligned}
& \text { codification }(\mathrm{A}): \text { Low }=0 ; \text { medium }=1 ; \operatorname{high}=2 ; \\
& \text { codification }(\mathrm{B}): \text { Low }=1 ; \text { medium }=3 ; \operatorname{high}=9 .
\end{aligned}
$$

Synthesizing by the arithmetic mean operator the results obtained in each sample, sample MMMML achieves a higher score than sample HMLLL with the first codification (A) and a lower score with the second codification (B) (see figure 6).

We have to remember that the numerical codification and elaboration of ordinal data is an improper operation (Roberts 1979, Franceschini and Romano 1999).

Figure 6 shows the behaviour of the arithmetic mean obtained by the codifications $\mathrm{A}$ and $\mathrm{B}$ applied to the space $P^{\prime}$. These two different codifications sometimes give rise to contradictory results.

An alternative approach can be based on the use of synthesis indexes which only utilize the ordinal properties of the measurement scale of variable $X$, for example, the sample median. Figure 7 shows a graphical comparison between the two lines according to the median index applied to the samples of table 1 near here (Clifford 1959).

Even though the median is an operator coherent with ordinal scales property, its efficacy is deeply conditioned by the dimension of the sample to which it is applied and by the number of levels of the evaluation scale. Furthermore, the median is insensitive to outliers. For discrete data that take on relatively few values, quite different patterns of data can give the same result (Chakraborty and Chaudhuri 1999, Montgomery 2001). In the example at hand, very different samples give the same median value. 


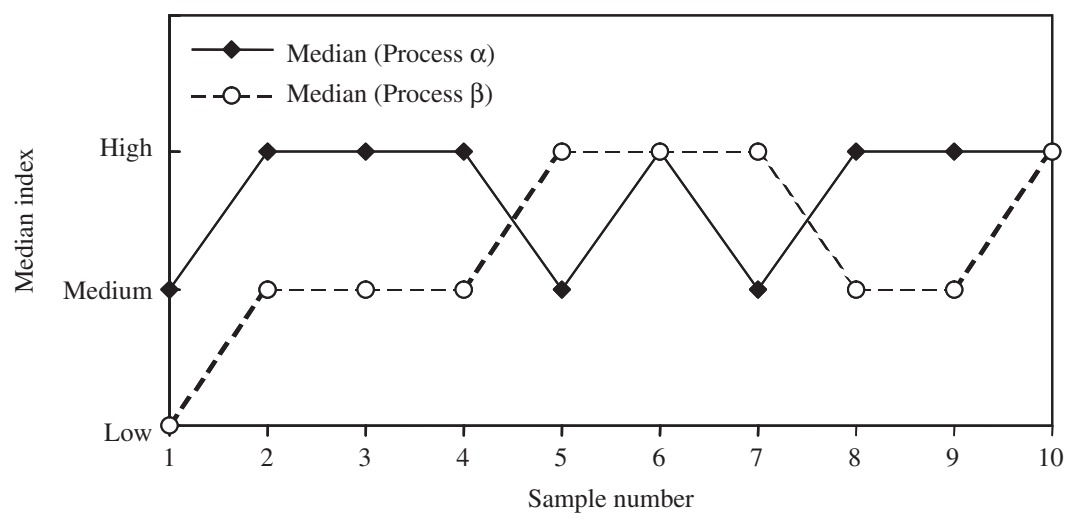

Figure 7. Median index of the two manufacturing processes (see table 1).

Limitations highlighted for the median may be overcome by using a new index based on the total ordering of $P^{\prime}$. In effect, the median is only one of the possible aggregation 'logics' that can be expressed by a map function $\varphi$.

A new ordering on $P^{\prime}$ can be constructed by adopting a specific map function $\varphi$ (Franceschini et al. 2005). Referring to data in table 1, suppose that, according to the peculiarities of our manufacturing process, we define an index according to which any sample including a 'low' rating is considered worse than any sample that does not include it. So, for example, HHHHL is worse than MMMMM. Samples which do not include any 'low' are ordered according to the already existing ranking on $P^{\prime}$.

This situation occurs in many contexts, in which the worst outcome is to be avoided. Consider examples involving safety issues (a few minor conjures are more acceptable than a single fatality), or cases in which a product falling into the worst category must be completely scrapped and thus provides significant financial losses to its producer.

This 'no low' criterion can be applied by using the map function $\varphi$ reported in figure 8 . The resulting totally ordered set $Q$ (chain) is the outcome of its application.

The map function of figure 8 has been applied to data in table 1 near here for the two analysed lines. The graphical behaviours of the two processes $\alpha$ and $\beta$ are reported in figure 9.

The graphical comparison between the two processes shows that process $\beta$ presents a synthesis index superior or equal to the one of process $\alpha$ in most of samples (seven out of 10); moreover, for the process $\beta$, the value 'low' has been got only twice and for the process $\alpha$ such a value has a very high frequency $(50 \%)$. This result is in stack contrast to that obtained using the median operator (see figure 7). Comparing samples 2, 3 and 4, process $\alpha$ exceeds process $\beta$ when using median operator; the opposite happens when using the 'no low' criterion.

\section{Synthesis maps for multivariate ordinal variable}

Lattice theory can be also applied in problems dealing with the 'synthesis' of components of a multivariate ordinal variable. 


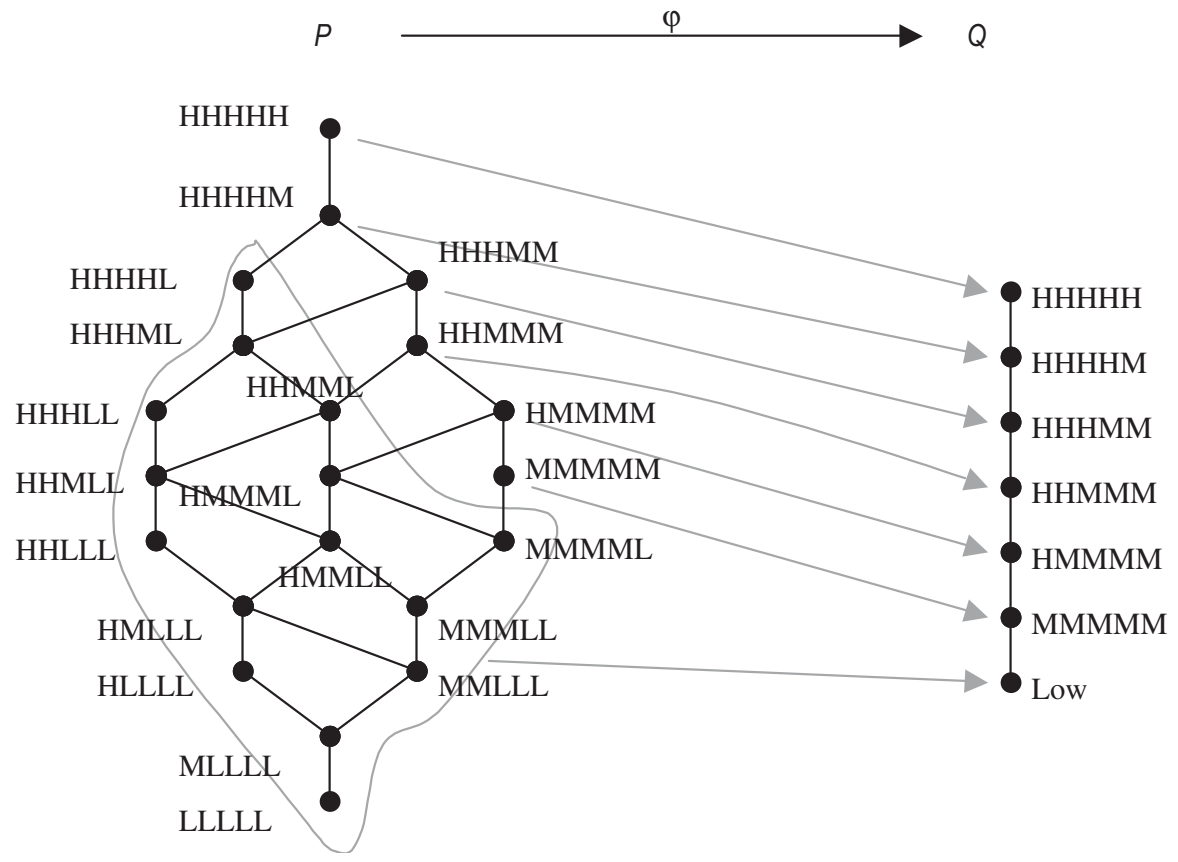

Figure 8. Hasse diagram for the combination space $P^{\prime}$ of a three categories (L, low; $\mathrm{M}$, medium; $\mathrm{H}$, high) ordinal variable $X$ (sample size, 5). The monotone map function $\varphi$ maps from $P^{\prime}$ to the chain $Q$ according to the 'no-low' criterion.

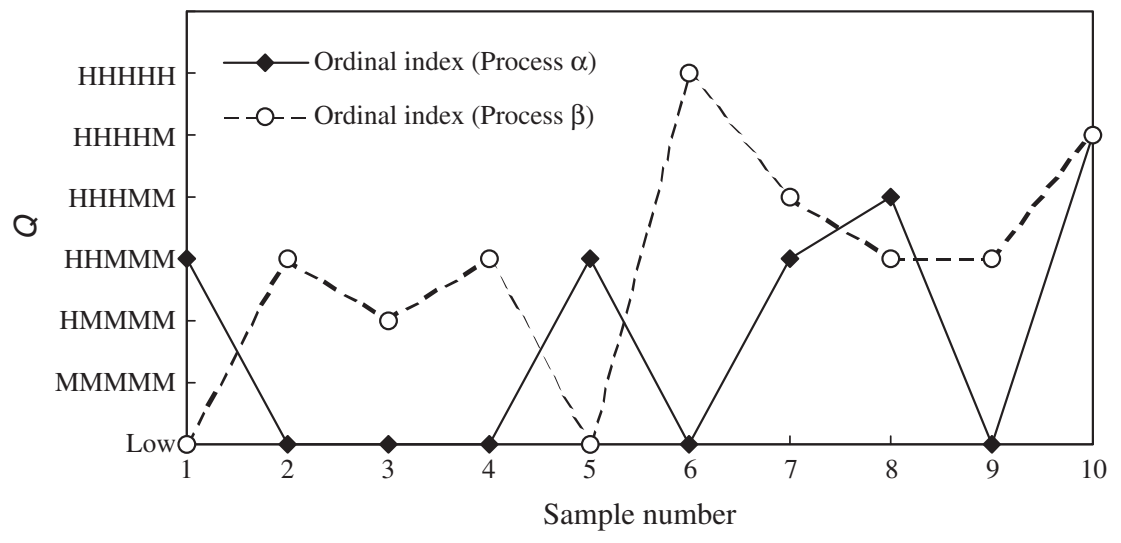

Figure 9. Ordinal Indexes for the two manufacturing processes.

This problem can be approached as follows:

1. Construction of an appropriate map function for aggregating the variable components; such a function must be defined on the basis of the specific characteristics of the process and without introducing improper (numerical) codifications.

2. Representation by a Hasse diagram. 


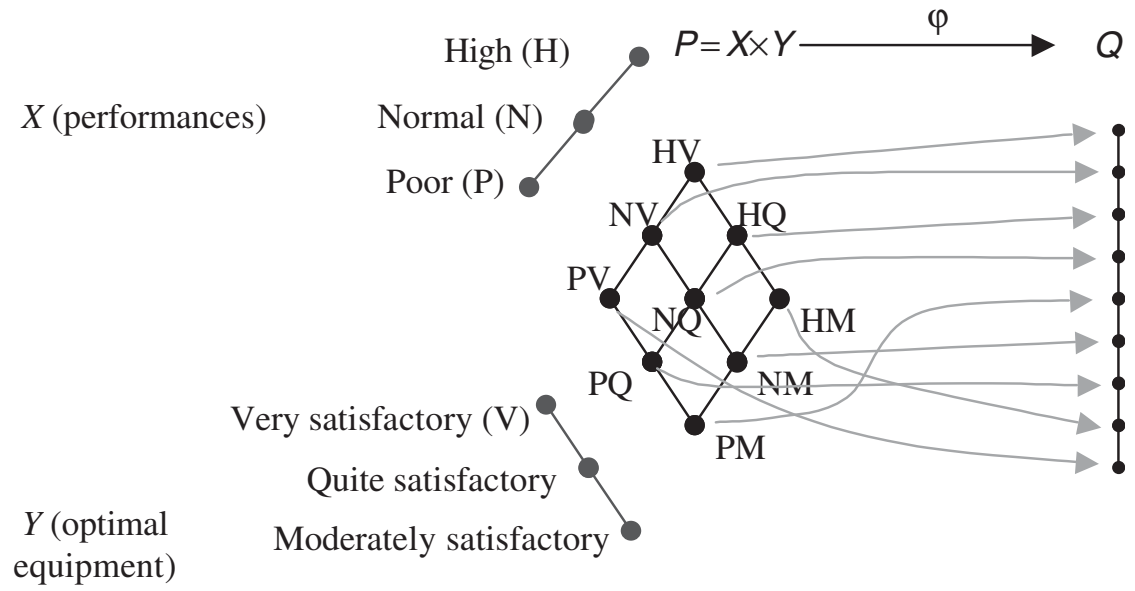

Figure 10. Non-order-preserving map function for a customer satisfaction survey (P, poor; $\mathrm{N}$, normal; $\mathrm{H}$, high; $\mathrm{M}$, moderately satisfactory; $\mathrm{Q}$, quite satisfactory; $\mathrm{V}$, very satisfactory).

3. Check of mathematical properties of the new ordinal variable defined in the map function co-domain.

4. Analysis of results.

Let us consider, for example, the following application case. A firm in the automotive field decided to analyse customer satisfaction by means of a survey. Each customer was asked to evaluate conjointly the performance and the satisfaction about the optional equipment (electric overdrive unit, air conditioning, compact disc player, etc.) of her/his new car on a three-category ordinal scale. The performance might be judged as 'poor', 'normal', or 'high' whereas the optional equipment is evaluated as 'moderately satisfactory', 'quite satisfactory' and 'very satisfactory'.

Some problems can arise considering conjointly these two ordinal variables. It seems reasonable that a car with poor performances and moderately satisfactory optional equipment represents the worst case. However, taking into account also the price of the car we can say that very satisfactory optional equipment accomplished by poor performance is disproportionate and also expensive. In other words, if a customer buys a cheap car with poor performance, perhaps he does not want many options in order to avoid an unnecessary expense. On the other hand, a car with high performance is very expensive, thus a moderately satisfactory judgement about the optional equipment involves poor satisfaction for the customer. Let us consider the two-dimensional ordinal variable $(X, Y)$ representing judgements of the customers about the performances and optional equipment of their cars. A 'possible' map function that could be applied to the set of all possible outcomes of the bivariate variable is shown in figure 10 .

The used map function allows interpreting the ordering criterion without resorting to any arbitrary numerical codification of scale levels.

Let us consider the data collected during 6 months on samples of 800 customers, summarised by the relative frequency distributions of a two-dimensional ordinal variable $(X, Y)$ as shown in table 2 near here (Bassotto et al. 2005). 
Table 2. Relative frequency values of a two-dimensional ordinal variable $(X, Y)$ (see figure 10). $(X, Y)$ provides customer evaluations of the performances and options of a new car. Data refer to samples of 800 customers interviewed during 6 months $(\mathrm{P}$, poor; $\mathrm{N}$, normal;

$\mathrm{H}$, high; $\mathrm{M}$, moderately satisfactory; $\mathrm{Q}$, quite satisfactory; $\mathrm{V}$, very satisfactory).

1st month 2nd month 3rd month 4th month 5th month 6th month

$(X, Y)$ Ranking in $Q$ frequency frequency frequency frequency frequency frequency

\begin{tabular}{lccccccc}
\hline HV & 1 & 0.03 & 0.02 & 0.08 & 0.17 & 0.08 & 0.03 \\
NV & 2 & 0.25 & 0.27 & 0.10 & 0.03 & 0.11 & 0.24 \\
HQ & 3 & 0.28 & 0.28 & 0.11 & 0.18 & 0.20 & 0.25 \\
NQ & 4 & 0.00 & 0.00 & 0.08 & 0.21 & 0.09 & 0.00 \\
PM & 5 & 0.04 & 0.03 & 0.25 & 0.14 & 0.13 & 0.05 \\
NM & 6 & 0.11 & 0.12 & 0.18 & 0.14 & 0.04 & 0.12 \\
PQ & 7 & 0.20 & 0.18 & 0.08 & 0.02 & 0.16 & 0.22 \\
HM & 8 & 0.09 & 0.09 & 0.11 & 0.03 & 0.13 & 0.08 \\
PV & 9 & 0.00 & 0.01 & 0.02 & 0.08 & 0.05 & 0.01 \\
& Total & 1.00 & 1.00 & 1.00 & 1.00 & 1.00 & 1.00 \\
\hline
\end{tabular}

Table 3. Month synthesis values for the samples of table 2 .

Data refer to samples of customers interviewed during 6 months.

\begin{tabular}{lc}
\hline Month & Sample synthesis index \\
\hline 1st month & HQ \\
2nd month & HQ \\
3rd month & PM \\
4th month & NQ \\
5th month & PM \\
6th month & HQ \\
\hline
\end{tabular}

For each month, a synthesis index consistent with ordinal properties of the variable $(X, Y)$ is calculated (see section 3). Table 3 shows the obtained results.

Data reported in table 3 can be represented by a two-dimensional diagram: 'optimal equipment' versus 'performance'. The sequence over time of the synthesis index values (index path) can be represented by oriented arcs drawn through the quadrants of that diagram. Figure 11 shows the map of the index path through quadrants during the 6 month observation.

The synthesis index of the new univariate variable (chain $Q$ ), obtained by ordering the two-dimensional space of the ordinal variable $(X, Y)$, can be considered as an 'ordinal index' able to interpret the trend of customer multiattribute preference. The diagram in figure 11 describes the path in the $X \times Y$ space, but does not give any indication about the evolution over time. To complete this information, figure 12 shows the behaviour over time of the index values for the samples analysed in the 6 months experimentation. Increasing or decreasing customer preference is observable on the nine-level ordinal scale described by the map function of figure 10 .

A numerical encoding of the two components of the bivariate variable $(X, Y)$ introduces additional properties (for example, interval properties) that could produce anomalous results. 


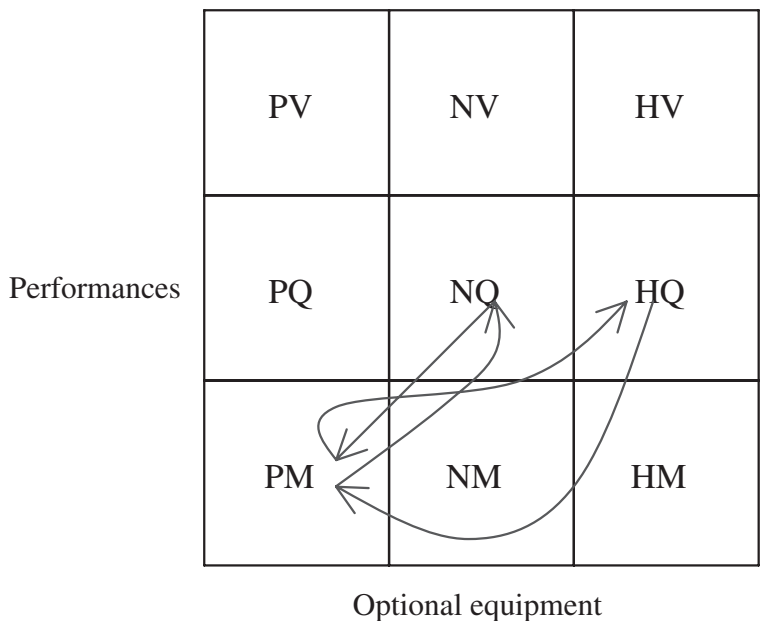

Figure 11. Path of the synthesis index of the univariate variable distribution obtained by ordering the two-dimensional space of the ordinal variable $(X, Y)$ (see figure 10). Data refer to samples of 800 customers interviewed during 6 months.

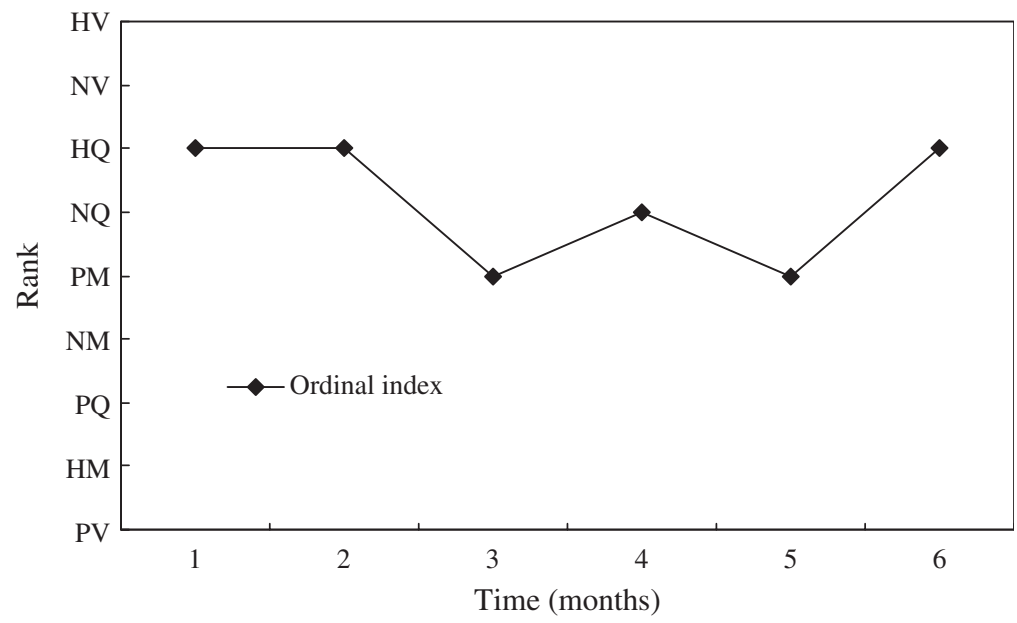

Figure 12. Behaviour over time of the synthesis index of the univariate variable distributions obtained by the ordering of the two-dimensional sample space of the ordinal variable $(X, Y)$ (see map function of figure 10). Data refer to samples of 800 customers interviewed during 6 months.

For example, let us consider the following encoding:

$$
P=0 ; \quad N=1 ; \quad H=2 ; \quad M=0 ; \quad Q=1 ; \quad V=2 .
$$

If we map by the arithmetic sum operator all possible outcomes of the bivariate variable $(X, Y)$ into a new ordered univariate variable, we observe that the outcome NQ achieves the same score as outcomes HM and PV. Adopting the quadratic sum operator it obtains a lower score. 
In general, other mapping functions can be applied without violating the original properties of the bivariate ordinal variable $(X, Y)$. That proposed is one of many, built according to our specific need. They can all be studied and analysed using the lattice theory approach (Tukey 1975, Barnett 1976).

\section{Conclusions}

Multidimensional ordinal structures are widely considered when dealing with ordinal variables in multidimensional problems both in engineering applications and in social/economics sciences.

This paper introduces a new methodology for the construction of synthesis indices based only on the ordinal proprieties of multidimensional ordinal structures. The algebraic theory of the ordered sets can be effectively applied to rank the sample space of a multivariate ordinal variable. Defining adequate functions (map functions) from finite sets into chains, a decision-maker can establish specific rankings in the multidimensional structures, namely, new ordinal indices.

The main characteristics of the proposed methodology are the following:

- The information about different components of a multidimensional structure can be summarized by a single ordinal non-numeric index defined on an ordered set.

- No numerical conversion of the ordinal scale levels is required.

- The ranking criterion is defined according to the 'physics' of the problem or to the objective of the decision-maker.

- The behaviour of the synthesis index can be detected and analysed over time.

- The variable sets can be described by a multidimensional graph (Hasse diagram), which facilitate the interpretation of the multidimensional ordinal structure.

Furthermore, the paper presents some application examples in which an organic comparison between the considered synthesis operators and the classical approaches is performed. It must be highlighted that traditional operators (such as, for example, the median) can be considered themselves particular examples of map functions.

The comparison shows that operators based on some arbitrary numerical conversions present contradictory results. On the contrary, the proposed method is coherent with the original properties of the ordinal scales.

Future developments of the research will regard the application of the lattice theory to multi-attribute decision-making models. The aim is the modelization, in terms of map functions, of criteria weights (Zopounidis and Doumpos 2003).

\section{Acknowledgements}

The authors wish to acknowledge the support of the Ministero dell'Istruzione, dell'Università e della Ricerca Scientifica (MIUR - ITALY) under grant no. 2001138489, year 2001. 'Metodi e modelli statistici per la valutazione della qualità e della Customer Satisfaction: aspetti oggettivi e soggettivi nell'ottimizzazione della qualità di beni e servisi'. Co-ordinator Professor A. Zanella. 


\section{References}

Agresti, A., Categorical Data Analysis, 2002 (Wiley: New York, NY).

Barnett, V., The ordering of multivariate data (with discussions). J. Roy. Statist. Soc., Ser. A, 1976, 139, 318-354.

Bassotto, C., D’Aprile, F., Davanzo, G., Franceschini, F., Maisano, D. and Spirito, F., Customer satisfaction: spunti per l'analisi in automatico di reclami o di lamentele su prodotti/servisi. Qualità, 2005, 1-2, 35-38 and 51-54.

Chakraborty, B. and Chaudhuri, P., A note on the robustness of multivariate medians. Stat. \& Prob. Let., 1999, 45, 269-276.

Clifford, P.C., Control charts without calculations. Indust. Qual. Cont., 1959, 15, 2-6.

Davey, B.A. and Priestley, H.A., Introduction to Lattices and Order, 1990 (Cambridge Mathematical Textbooks: Cambridge).

Donnellan, T., Lattice Theory, 1968 (Pergamon Press: Oxford).

Franceschini, F. and Galetto, M., A new approach for evaluation of risk priorities of failure modes in FMEA. Int. J. Prod. Res., 2001, 39, 2991-3002.

Franceschini, F., Galetto, M. and Varetto, M., Ordered samples control charts for ordinal variables. Qual. \& Rel. Eng. Int., 2005, 21, 177-195.

Franceschini, F. and Romano, D., Control chart for linguistic variables: A method based on the use of linguistic quantifiers. Int. J. Prod. Res., 1999, 37, 3791-3801.

Krantz, D.H., Luce, R.D., Suppes, P. and Tversky, A., Foundations of Measurement: Volume 1. Additive and Polynomial Representations, 1971 (Academic Press: New York).

Liu, R.Y., Parelius, J.M. and Singh, K., Multivariate analysis by data depth: Descriptive statistics, graphics and inference (with discussions). Ann. Statist., 1999, 27, 783-858.

Liu, R.Y. and Singh, K., A quality index based on data depth and multivariate rank tests. J. Amer. Statist. Assoc., 1993, 88, 257-260.

Montgomery, D.C., Introduction to Statistical Quality Control, 4th edn, 2001 (Wiley: New York, NY).

Moshkovich, H.M., Mechitov, A.I. and Olson, D.L., Ordinal judgments in multiattribute decision analysis. Euro. J. Op. Res., 2002, 137, 625-641.

Parasuraman, A., Zeithaml, V.A. and Berry, L.L., A conceptual model of service quality and its implications for future research. J. Market., 1985, 49, 41-50.

Roberts, F.S., Measurement theory with applications to decision making, utility and the social sciences. in Encyclopaedia of Mathematics and its Applications. Vol. 7, 1979 (AddisonWesley Publishing Company: New York).

Roy, B. and Bouyssou, D., Aide Multicritère à la decision: Méthodes et Cas, 1993 (Economica: Paris).

Taleb, H. and Limam, M., On fuzzy and probabilistic control charts. Int. J. Prod. Res., 2002, 40, 2849-2863.

Tukey, J.W., Mathematics and the picturing of data. In Proceedings of the International Congress of Mathematicians, edited by R.D. James, Vol. 2, pp. 523-531, Vancouver, 1975 (Canadian Mathematical Congress: Montreal, Canada).

Zopounidis, C. and Doumpos, M., Multicriteria classification and sorting methods: A literature review. Euro. J. Op. Res., 2002, 138, 229-246. 